et al. Early-onset absence epilepsy and paroxysmal dyskinesia. Epilepsia Oct 2002;43:1224-1229). (Reprints: Prof R Guerrini, Institute of Child Health and Great Ormond Street Hospital, London WC1N 2AP, UK).

COMMENT. Absence epilepsy and paroxysmal dyskinesias may coexist. The age at onset of absence seizures and $3 \mathrm{~Hz}$ spike-and-wave in this comorbid syndrome is unusually early, often in infancy.

\title{
RISK OF OVULATORY FAILURE WITH EPILEPSY
}

The association of ovulatory dysfunction with epilepsy and antiepileptic drugs (AEDs) was evaluated in women aged 18 to 40 years not receiving hormones recruited from the Stanford and Columbia Universities Comprehensive Epilepsy Centers and from other sources. Patients were followed for three menstrual cycles, a transvaginal ovarian ultrasound was obtained, and multiple endocrine and metabolic factors, including luteinizing hormone were sampled over 8 hours on days 2 to 5 of one cycle. Anovulatory cycles occurred in $10.5 \%$ of cycles in control patients without epilepsy (23), $14.3 \%$ of cycies with localization-related epilepsy (59 patients), and $27.1 \%$ of cycles with idiopathic (primary) generalized epilepsy (35 patients). At least one anovulatory cycle occurred in $38.1 \%$ of women with epilepsy who were taking valproate currently or within 3 years, and in $10.7 \%$ of non-valproate medicated patients. Risk factors for ovulatory dysfunction include idiopathic generalized epilepsy, exposure to valproate AED, high free testosterone, and reduced luteinizing hormone pulses. Patients with polycysticappearing ovaries ( $41 \%$ of those with idiopathic generalized epilepsy cf $16 \%$ women without epilepsy) are not at increased risk and may ovulate normally. (Morrell MJ, Giudice L, Flynn KL et al. Predictors of ovulatory failure in women with epilepsy. Ann Neurol December 2002;52:704-711). (Respond: Dr Martha J Morrell, The Neurological Institute, Department of Neurology, 710 West 168th Street, New York, NY 10032).

COMMENT. Women with idiopathic generalized epilepsy are at increased risk for ovarian dysfunction, anovulatory cycles, and polycystic-appearing ovaries. Those treated with valproate, a cytochrome P450 enzyme inhibiting AED, are at highest risk, and this AED has an additive adverse effect. Cytochrome P450 inducing AEDs (carbamazepine, phenytoin, phenobarbital), and AEDs with no effect on cP450 (lamotrigine, gabapentin) have no adverse additive effect on ovarian function. Ovarian function should be monitored in women with epilepsy. The anovulatory cycles may be the only sign of reproductive dysfunction.

\section{PERIODIC SYNDROMES}

\section{DIAGNOSIS OF CYCLIC VOMITING}

In a study at Children's Hospital, Ann Arbor, MI, and Children's Memorial Hospital, Chicago, designed primarily to compare cost-effectiveness of three methods of management of cyclic vomiting cases, one group of patients received an extensive diagnostic evaluation, a second was treated with empiric antimigraine drugs for 2 months, and a third an upper GI series with small-bowel follow-through (UGI-SBFT) plus empiric therapy. Cyclic vomiting is defined as $>3$ episodes of vomiting within a 3 month period, peak intensity averaging 6 emeses per hour, and intervals of normal health averaging 2 to 4 weeks. The most costeffective approach was the UGI-SBFT followed by antimigraine therapy. A CT scan before antimigraine therapy to rule out a brain tumor would be cost-saving, even 